\title{
Numerical Simulation of Supersonic Over-expanded Jet from 2-D Convergent-Divergent Nozzle
}

\section{Devendra Kumar Lohia ${ }^{1}$, Bholu Kumar ${ }^{2}$, Shantanu Srivastava ${ }^{2}$, H. K. Paliwal ${ }^{3}$}

${ }^{1}$ Department of Mechanical Engineering, Institute of Technology and Management, Gorakhpur 73209, India. ${ }^{2}$ Department of Mechanical Engineering, National Institute of Technology, Patna 800006, India.

${ }^{3}$ Department of Mechanical Engineering, Institute of Engineering and Technology, Lucknow 226021, India.

Received 19 July 2018; accepted 23 December 2018, available online 31 December 2018

\begin{abstract}
The present study focuses on the numerical investigation of the Mach 1.86 supersonic jet from the 2-D convergent-divergent nozzle for NPRs 4 to 6 with a step size of one, covering the over expanded and the near correct expansion conditions. The computational model is constructed into design modular of ANSYS 16.0 workbench. The structured grids are generated into the ICEM module of the ANSYS workbench and the fluid flow analysis, on the modelled part is done by FLUENT solver. The extent of jet mixing is identified by plotting non-dimensional total pressure, along the jet centerline, with respect to the non-dimensional distance downstream of the nozzle exit and along the jet centerline. Also the Mach contours are plotted for different NPRs indicating, the number of shock cells and the variation of the shock strength with respect the change in the NPR due to the prevailing adverse pressure gradient at the nozzle exit.
\end{abstract}

Keywords: Jet mixing, centerline pressure decay, Mach number, NPR, viscous activity.

\section{Introduction}

The study of supersonic jet has gained importance in the recent past due to its applicability in the wide variety of engineering applications beginning from household appliances to high tech rockets. In terms of academic interest, studies on jets have provided insight into the understanding of the dynamics of free shear layers and vortical structures. The jet may be defined as a pressure driven free share flow which exhibits the characteristics that its local width to the local axial distance bears a constant value, which is 8 for Mach numbers less than 0.2 . This ratio decreases with the increasing Mach numbers. The jet may also be defined as the fluid issuing from a nozzle into quiescent surrounding. The jet which is issuing such that the ambient surrounding fluid is at rest is known as submerged jet, as has been established in the open literature.

As the jet issues from the nozzle into the free environment, it slows down due to the stagnant ambient fluid, inducted into the jet field. The shear layer downstream of the nozzle exit, develops roll-up structures and ring vortices, due to the shearing action between the two layers of fluid. These roll-up structures are typically known as vortical structures in the open literature. The vortical structures are essentially significant in bringing the ambient fluid mass from the surrounding environment into the jet field. This phenomenon is known as entrainment [1].

As the jet further travels downstream of the nozzle exit, these vortical structures travel in the transverse direction of the jet, right up to the jet centerline. The vortical structures generated within the shear layer are also termed as coherent structures [2].

It has been established in the open literature that, for an early and rapid jet mixing there should be proper proportion of the large scale and small scale vortices present in the jet field. However, finding out this proper proportion of large and small scale vortices is almost next to impossible, especially for a turbulent velocity field like a supersonic free jet. Thus, an indirect method which might be used for the quantification of the jet mixing is the rapid decay of the jet centerline velocity or the core length of the jet. This demonstrates that, a jet with shorter core length will comparatively have better and enhanced mixing, to a similar jet with a longer core length $[3,4,5]$.

Due to the complexity of flow, jets are mostly studied experimentally. These complexities arise due to the entrainment of the jets, large perturbations present at the high Reynolds number and strong wave interactions in jets exiting at supersonic Mach numbers from typical convergent-divergent nozzles. However, there are some researchers who did computational study on the mixing characteristics of a supersonic jet. An account of computational investigations on supersonic jet is the following.

Launder and Spalding [6] investigated the efficacy of $k-\varepsilon$ turbulence model for the simulation of jet from a convergent-divergent nozzle. It was found that, the $\kappa-\varepsilon$ turbulence model is capable of predicting the freeshear flow phenomena without adjustments of constants 
and functions. However, the model can only accurately predict the features for low Reynolds number flows. Dash et. al. [7] upgraded the existing $\kappa-\varepsilon$ turbulence model by introducing the unification of vortex-stretching and compressible-dissipation extensions in order to put the results in agreement with the existing fluid dynamic data. It was reported that, utilization of this turbulence model in a simple parabolic solver combined with a Rayleigh instability analysis provides the basis for predicting noise associated with Mach emission. Tam and Thies [8] investigated the $\kappa-\varepsilon$ turbulence model for supersonic jets. However, the investigation and results were limited to the simulation of velocity profiles and the Mach number profile, downstream of the nozzle exit. Evgenevna et. al. [9] evaluated the prediction capabilities of various twoparameter differential turbulence models. The work was confined to only correctly expanded supersonic jets and it was found that, $\mathrm{k}-\varepsilon$ realizable and transition SST turbulence models, showed the best results.

So far, it has been demonstrated that the k- $\varepsilon$ realizable turbulence model and the transition SST turbulence model show promising results for the simulation of free jets from convergent-divergent nozzles. However, the computational cost and resources requirement involved for more sophisticated turbulence models were exorbitantly high. The present investigation aims at finding out the mixing extent and simulation of the Mach 1.86 jet from the convergent-divergent nozzle, at overexpansion and near correct-expansion conditions (correct expansion for Mach 1.86 is at NPR 6.29), with a $\mathrm{k}-\varepsilon$ realizable turbulence model through a commercial software package, without requiring High Performance Computation Facility. The study aspires to develop a computationally economic method for the simulation of high speed flows without the requirement of sophisticated computational facility. However, care is taken so as to make the accuracy of the computations within acceptable accuracy limits, close to the established experimental results. The comparison of the results is achieved with the experimental results of Shantanu and Rathakrishnan [10]. In the present work, a computational study is performed with the 2-D axisymmetric uncontrolled jets, (3-D being computationally costly). The NPR of the jet is varied from 4 to 6 with a step size of one. The mixing characteristics of over expanded uncontrolled supersonic jet is studied by plotting the non-dimensional total pressure with respect to the non-dimensional distance, downstream of the nozzle exit, along the jet centerline. In addition to this, Mach contours are also plotted at different NPRs for the qualitative aspects of the study and visualization of other features of the jet including the barrel shock, the compression waves and the expansion fans.

\section{Methodology}

In the present study, an axisymmetric two dimensional computational model is constructed in ANSYS software of version 16.0. The domain extends to about 30D from the nozzle exit, along the jet centerline and 15D along the transverse direction to the jet axis, where $\mathrm{D}$ is the diameter of the nozzle exit plane geometry. The grid is generated using the ICEM module of the ANSYS workbench 16.0 and the flow of the jet, in two dimensions, is analyzed in FLUENT.

\subsection{Numerical Domain}

The sketch of computational domain for the axisymmetric model, is as shown in Fig. 1. The convergent-divergent nozzle is constructed with convergent angle of $15^{\circ}$ and divergent angle of $7^{0}$. This C-D nozzle is designed to deliver a supersonic jet of Mach 1.86. The diameter (D) of the nozzle is found to be $12.28 \mathrm{~mm}$ with reference to the nozzle throat diameter of $10 \mathrm{~mm}$ which is evaluated from the Area-Mach number relationship, for Mach 1.86 jet [1]. The computational domain extends to about 30D along the jet axis and to about $15 \mathrm{D}$ in the transverse direction to the jet axis.

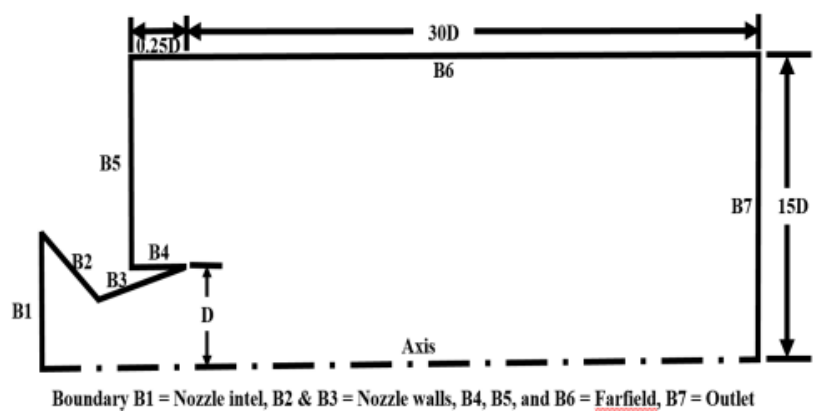

Fig. 1 Schematic diagram of the computational domain

\subsection{Boundary conditions}

Nozzle Inlet (B1): At the nozzle inlet boundary (B1), the pressure inlet boundary condition is specified. The pressure at inlet of the nozzle is varied such that, the NPR varies 4 to 6 with a step size of one. A constant temperature of $300 \mathrm{~K}$ is prescribed.

Nozzle walls (B2 \& B3): The wall boundary condition is specified for the nozzle walls B2 and B3. The walls of nozzle are assumed to be adiabatic with no slip condition.

Pressure Far-field (B4, B5, and B6): The pressure far-field boundary condition is specified for the boundaries B4, B5, and B6, of the flow domain.

Pressure Outlet: The pressure outlet boundary condition is specified for the domain boundary B7.

Axis: This boundary represents the centerline of the jet. For the present axisymmetric problem, an axis boundary condition has been specified as shown in Fig. 1.

\subsection{Mesh generation}


The 2-D quadrilateral structured grids were generated, as shown in Fig. 2, with grid sizes of 56016, 87237, 114274, 126036, 154680, 223581, and 349117. The grid independence test for the above grid sizes is as shown in Fig. 3. The Mach number of the jet, issuing from the nozzle, along the jet centerline is computed at the different spatial locations and the grid independence test is conducted for all the above seven cases of grid sizes. The results are found to be independent of the grid size at all spatial locations along the jet centerline for the grid size of at least 223581 . Thus, the grid size of 223581 is adopted for the complete mixing investigation of the jet. The maximum skewness of 0.5 and a maximum aspect ratio of 2.38 are reported for the present grid size of 223581. The plot of Mach number along the jet centerline is shown in the Fig. 3, which clearly demonstrates the grid independence of the cells, at and above the grid size of 223581.

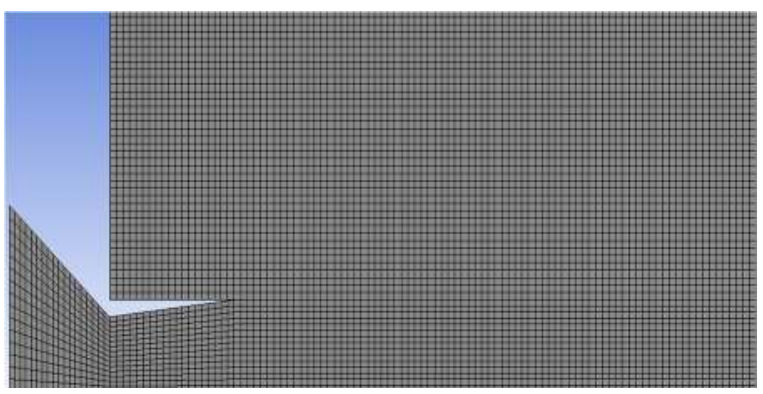

Fig. 2 Computational Grid

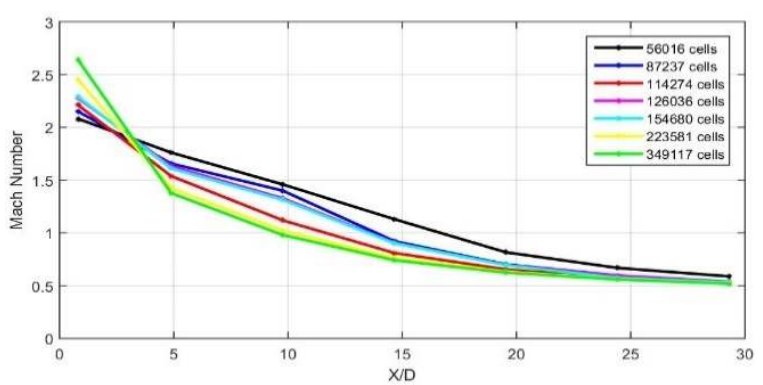

Fig. 3 Grid Independence test: A plot of Mach number along jet centerline

\subsection{Numerical Procedure}

The governing equations involving conservations of mass, momentum and energy are solved using FLUENT, which employs Finite Volume Method. The steady-state equations have been solved with double precision accuracy, an approach to mitigate the effect of typical round-off errors. A second order upwind scheme is used for modelling the flow, the turbulent kinetic energy and the turbulent dissipation rate. The governing equations for fluid flow are the following.

\subsubsection{Continuity Equation (Conservation of Mass)}

The two dimensional continuity equation for compressible flow of jet can be expressed as $[11,12]$

$$
\frac{\partial \rho}{\partial t}+\nabla \cdot(\rho \stackrel{\mathrm{r}}{\rho})=0
$$

Where, $\stackrel{\mathrm{r}}{V}=u \stackrel{\mathrm{r}}{\mathrm{i}}+\underset{\mathrm{r}}{\mathrm{r}}, \mathrm{u}$ and $\mathrm{v}$ are the components of velocity along the $\mathrm{x}$ and $\mathrm{y}$ direction respectively and $\rho=\rho(x, y)$ is the density of air.

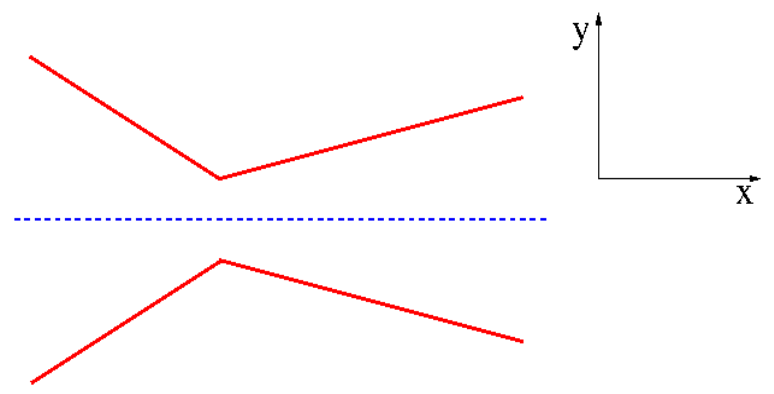

Fig. 4 Cartoon showing direction of jet propagation

\subsubsection{Momentum Equation}

The $\mathrm{x}$-component of momentum equation can be written as $[11,12]$

$$
\frac{\partial(\rho u)}{\partial t}+\nabla \cdot(\rho u \stackrel{\mathrm{r}}{V})=\frac{-\partial p}{\partial x}+\frac{\tau_{x x}}{\partial x}+\frac{\tau_{y x}}{\partial y}+\rho f_{x}
$$

Similarly, the y-component of the momentum can be written as

$$
\frac{\partial(\rho v)}{\partial t}+\nabla \cdot(\rho v \stackrel{\mathrm{r}}{V})=\frac{-\partial p}{\partial x}+\frac{\tau_{x y}}{\partial x}+\frac{\tau_{y y}}{\partial y}+\rho f_{y}
$$

Where, $\tau_{x x \text { and }} \tau_{y y}$ denotes the shear stresses in the direction of $\mathrm{X}$ and $\mathrm{Y}$ respectively.

$\tau_{y x \text { and }} \tau_{y x}$ denotes the normal stresses in the direction of $\mathrm{X}$ and $\mathrm{Y}$ and acting normal to the direction of $\mathrm{Y}$ and $\mathrm{X}$ respectively.

$f_{x}$ and $f_{y}$ are the body forces per unit mass along $\mathrm{X}$ and $\mathrm{Y}$ direction respectively.

\subsubsection{Energy Equation}

The energy equation is the law of conservation of energy, which states that the rate of change of energy inside the fluid element is equal to the sum of net flux of heat into the element and the rate of work done on the element due to body and surface forces. The energy equation for steady compressible flow can be mathematically expressed as; $[11,12]$

$$
\begin{aligned}
& \frac{\partial}{\partial}\left[\rho\left(e+\frac{V^{2}}{2}\right)\right]+\nabla \cdot\left[\rho\left(e+\frac{V^{2}}{2} V\right)\right]=\rho q+\frac{\partial}{\partial x}\left(k \frac{\partial T}{\partial x}\right)+\frac{\partial}{\partial y}\left(k \frac{\partial T}{\partial y}\right) \frac{\partial(u p)}{\partial x} \frac{\partial(p)}{\partial y}+ \\
& \frac{\partial\left(u \tau_{x x}\right)}{\partial x}+\frac{\partial\left(u \tau_{y x}\right)}{\partial y}+\frac{\partial\left(v \tau_{x y}\right)}{\partial x}+\frac{\partial\left(v \tau_{y y}\right)}{\partial y}+\rho f V \\
& \operatorname{unr}
\end{aligned}
$$

Where, $q$ and e , k, p are the source term per unit mass, the internal energy of the fluid element, thermal conductivity of the pressure respectively.

\subsubsection{Turbulence Model}


Realizable $\mathrm{k}-\varepsilon$ turbulence model is applied in the present investigation to capture the effects of turbulence, so as to quantify the mixing characteristics of the jet. It was envisaged that, the k-epsilon model would improve the mixing-length model and in addition, will find an alternative to algebraically prescribed turbulent length scales in moderate to high complexity flows. The transport equations for $k$ (turbulent kinetic energy) and $\varepsilon$ (turbulent dissipation) in the Realizable $k-\varepsilon$ turbulent model are $[13,14]$;

$\frac{\partial}{\partial t}(\rho k)+\frac{\partial}{\partial x_{j}}\left(\rho k u_{j}\right)=\frac{\partial}{\partial x_{j}}\left[\left(\mu+\frac{\mu_{t}}{\sigma_{k}}\right) \frac{\partial k}{\partial x_{j}}\right]+P_{k}+P_{b}-\rho \varepsilon-Y_{M}+S_{k}$

$\frac{\partial}{\partial}(\rho \epsilon)+\frac{\partial}{\partial x_{j}}\left(\rho u_{j}\right)=\frac{\partial}{\partial x_{j}}\left[\left(\mu+\frac{\mu}{\sigma_{\epsilon}}\right) \frac{\partial \epsilon}{\partial x_{j}}\right]+\rho C_{1} S_{\epsilon}-\rho C_{2} \frac{\epsilon^{2}}{k+\sqrt{\vartheta \epsilon}}+C_{\epsilon} \frac{\epsilon}{k} C_{3 \epsilon} P_{b}+S_{\epsilon}$

\section{Where}

$\mu$ and $\mu_{t}$ denotes the coefficient of dynamic and eddy viscosity respectively.

$\sigma_{k}$ and $\sigma_{\epsilon}$ represents the turbulent Prandtl numbers for $\mathrm{k}$ and $\epsilon$ respectively.

In these equations, $P_{k}$ represents the generation of turbulence kinetic energy due to the mean velocity gradients, and $P_{b}$ is the generation of turbulence kinetic energy due to buoyancy.

$\mathrm{Y}_{\mathrm{M}}$ represents the contribution of fluctuating dilatation in compressible turbulence to overall dissipation rate.

$S_{k}$ and $S_{\epsilon}$ are the user defined source terms.

Now, the model constant $\mathrm{C}_{1}$ can be evaluated as;

$C_{1}=\max \left[0.43, \frac{\eta}{\eta+5}\right], \eta=S \frac{k}{\epsilon}, S=\sqrt{2 S_{i j} S_{i j}}$

Where, $S$ and $S_{i j}$ denotes the strain tensor and mean strain rate respectively.

\section{Modelling Turbulent Viscosity}

$$
\mu_{t}=\rho C_{\mu} \frac{k^{2}}{\epsilon}
$$

Where,

$$
\begin{gathered}
C_{\mu}=\frac{1}{A_{0}+A_{s} \frac{K U^{*}}{\epsilon}}, U^{*}=\sqrt{S_{i j} S_{i j}+\Omega_{i j}^{9_{0} g_{i j}^{0}}} \\
\Omega_{i j}^{g_{0}}=\Omega_{i j}-2 \epsilon_{i j k} \omega_{k}, \text { and } \Omega_{i j}=\bar{\Omega}_{i j}-\epsilon_{i j k} \omega_{k}
\end{gathered}
$$

Where $\Omega_{i j}$ is the mean rate-of-rotation tensor viewed in a rotating reference frame with the angular velocity $\omega_{k}$. The model constants $A_{0}$ and $A_{s}$ are given by:

$$
A_{0}=4.04, A_{s}=\sqrt{6} \cos \varnothing, \varnothing=\frac{1}{3} \cos ^{-1}(\sqrt{6} W) \text {, }
$$

and $W=\frac{S_{i j} S_{j k} S_{k i}}{S^{z}}, \xi=\sqrt{S_{i j} S_{i j}}, S_{i j}=\frac{1}{2}\left(\frac{\partial u_{j}}{\partial x_{i}}+\frac{\partial u_{i}}{\partial x_{j}}\right)$

Model constants

$$
C_{1 \epsilon}=1.44, C_{2}=1.9, \sigma_{k}=1.0, \sigma_{\epsilon}=1.2
$$

\subsection{Numerical Simulation}

The numerical simulations were carried out in the FLUENT solver of ANSYS software of version 16.0. The density-based steady solver is adopted for the numerical simulations, as the jet in the present investigation is compressible with Mach number 1.86. The implicit scheme and the convergence criteria of $1 \mathrm{e}-05$ are chosen for the convergence of the solutions. The computational economy is achieved by exploiting the symmetry of the nozzle, using axisymmetric formulation for the entire computational domain, as shown in Fig. 1.

\subsection{Computational Validation}

In the present study, CFD validation is done with the experimental results of Shantanu et al. [10] at NPR 5, as shown in the Fig. 5.

The centerline pressure decay plot of Mach 1.86 jet at NPR 5, is shown in Fig. 5. The figure shows a quantitative comparison between the experimental and simulation results, plotted as centerline pressure decay at NPR 5.

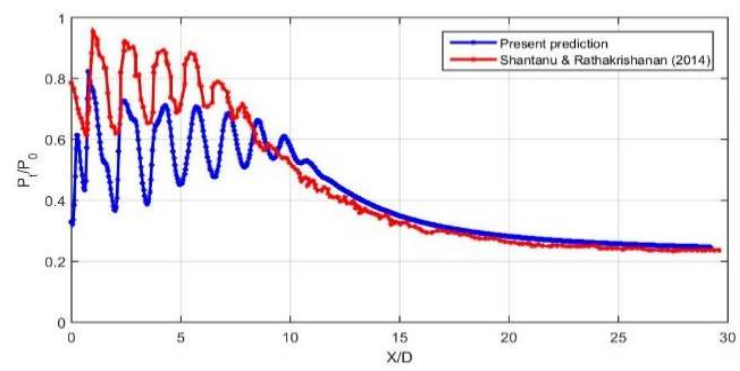

Fig. 5 Validation of present computation work with experiment results of Shantanu et al. (2014)

It was found that almost same number of shock cells are found in both the investigations viz., experimental and numerical. It is seen that there is a slight phase difference between the centerline pressure decay plots obtained experimentally and numerically. This could be attributed to the inability of the pitot probe to take measurements precisely from the origin point, due to physical interference of the probe with the physical model. In addition to this, the physical interference of the pitot probe leads to the generation of additional shocks in the flow field during experimentation. Due to the formation of shocks at the nozzle exit, the computational results show momentarily deceleration up to a distance of about $0.5 \mathrm{D}$ from the nozzle exit, which is otherwise not the case during experimentation. Thus, it is observed that, there would always be slight difference between the computational and experimental results due to the ability of computers to create an ideal experimental set up, which is not possible with physical experimentation. The flow accelerates thereafter due to the cumulative effect of the expansion fans and the relaxation offered by the large space of free environment.

\section{Results and Discussion}

It has been established in the open literature that, for efficient mixing of the jet there must be a proper proportion 
of large scale vortices and small scale vortices. However, finding out and maintaining this proper proportion of large scale and small scale vortices is next to impossible. Due to this reason there is no direct means to estimate the mixing of jet. However, the significant quantification of jet mixing can be achieved by the estimation of centerline pressure decay, right from the nozzle exit and along the jet centerline. The core length of the jet, which is defined as the axial distance along the jet centerline up to which the velocity of the jet remains supersonic is a direct indication of the extent of jet mixing. A shorter core length will have rapid mixing of the jet, compared to a longer jet core. The characteristics decay zone begins after the jet core. A sharp characteristic decay indicates rapid mixing and the ability of the jet attain self-similar profile rapidly. Jet core is the zone of supersonic axial velocity (nozzle exit velocity) along the jet axis. The centerline pressure decay is a plot of non-dimensional total pressure variation, with respect to the non-dimensional distance along the jet centerline. The total pressure is made non-dimensional by dividing it with the upstream stagnation pressure. Whereas, the distance along the jet centerline is made non-dimensional by dividing it with the diameter of the nozzle exit plane.

The centerline pressure decay for the Mach 1.86 jet at NPR 4 is shown in Fig. 6. At this NPR, the jet becomes over expanded with an overexpansion level of about $36.5 \%$. There is adverse pressure gradient at the nozzle exit. It is seen that, there are four prominent shock cells present downstream of the nozzle exit. The jet core length extends to about 5D. The characteristic decay zone, which extends after the potential core region extends from about $5 \mathrm{D}$ to $20 \mathrm{D}$, along the jet centerline. Beyond $\mathrm{X} / \mathrm{D}=20$, the jet attains self-similar profile, which is evident from the flat nature of the centerline pressure decay variation beyond $\mathrm{X} / \mathrm{D}=20$.

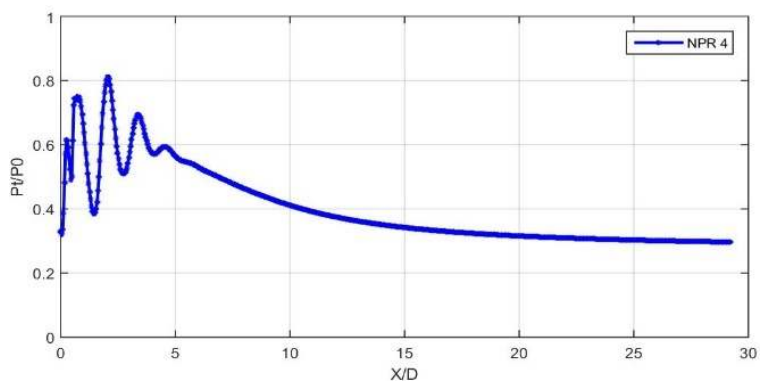

Fig. 6 Centerline pressure decay of Mach 1.86 uncontrolled jet at NPR 4

The centerline pressure decay plot for the Mach 1.86 jet at NPR 5 is shown in Fig. 7. At this NPR, the jet becomes over expanded with an overexpansion level of about $21 \%$. The flow experiences adverse pressure gradient at the nozzle exit, however, the level is reduced compared to that at NPR 4. It is seen that, there are seven prominent shock cells present downstream of the nozzle exit.

This is also evident from the Mach contour plots of the jet, as shown in Fig. 11. The jet core length extends to about 11D. The characteristic decay zone, extends from about $11 \mathrm{D}$ to $20 \mathrm{D}$, along the jet centerline. It is interesting to see that, the characteristic decay of the jet exhibits sharp nature. Thus, it might be inferred from the centerline pressure decay plot that, with a slight decrease in the overexpansion level, the jet shows faster mixing compared to that at lower NPR. The jet attains self-similar profile beyond $X / D=20$, which is evident from the flat nature of the centerline pressure decay variation beyond $X / D=20$.

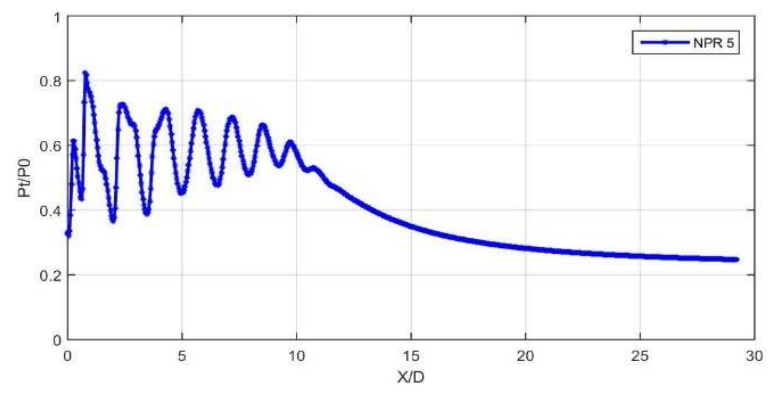

Fig. 7 Centerline pressure decay of Mach 1.86 uncontrolled jet at NPR 5

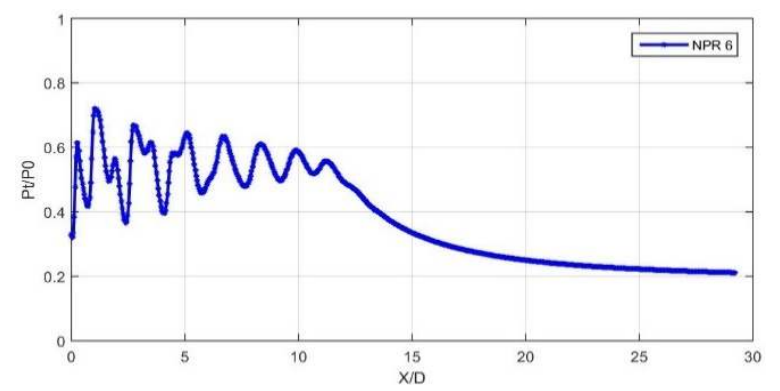

Fig. 8 Centerline pressure decay of Mach 1.86 uncontrolled jet at NPR 6

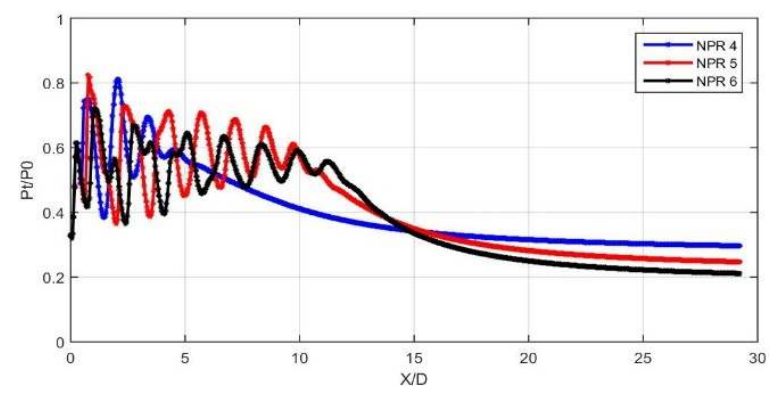

Fig. 9 Centerline pressure decay of Mach 1.86 uncontrolled jet with variation in NPR

The centerline pressure decay plot for the Mach 1.86 jet at NPR 6 is shown in Fig. 8. At this NPR, the jet is near the correct expansion with a marginal overexpansion level of about $5 \%$. The flow experiences adverse pressure gradient at the nozzle exit, however, the level is drastically reduced compared to that at NPR 4. It is seen that, there are eight prominent shock cells present downstream of the nozzle exit. This is also evident from the Mach contour plots of the jet, as shown in Fig. 11. The jet core length extends to about 12D. The characteristic decay zone, extends from about $12 \mathrm{D}$ to $20 \mathrm{D}$, along the jet centerline. It is interesting to see that, the characteristic decay of the jet exhibits sharp nature. Thus, it might be inferred from the centerline pressure decay plot that, with a slight decrease in the overexpansion level, the jet shows faster mixing compared to that at lower NPR. The jet attains self-similar profile 
beyond $X / D=20$, which is evident from the flat nature of the centerline pressure decay variation beyond $\mathrm{X} / \mathrm{D}=20$.

The comparative plots of centerline pressure decay and Mach number at different NPRs (4, 5 and 6) are shown in Fig. 9 and Fig. 10. It is evident from the centerline pressure decay plots that the mixing ability of the jet is strongly affected by the level of expansion at the nozzle exit. It is seen that the mixing extent improves with the increase in the NPR. However, the present study is focused on the mixing characteristics of the jet for the over expanded and near correct expansion conditions.

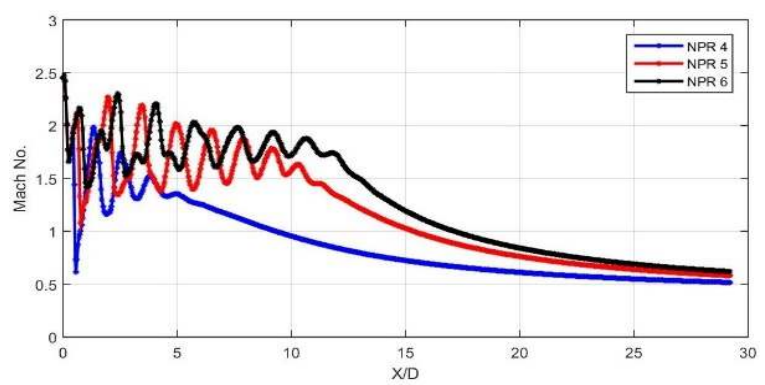

Fig. 10 Variation of Mach number in downstream of the jet with different NPRs

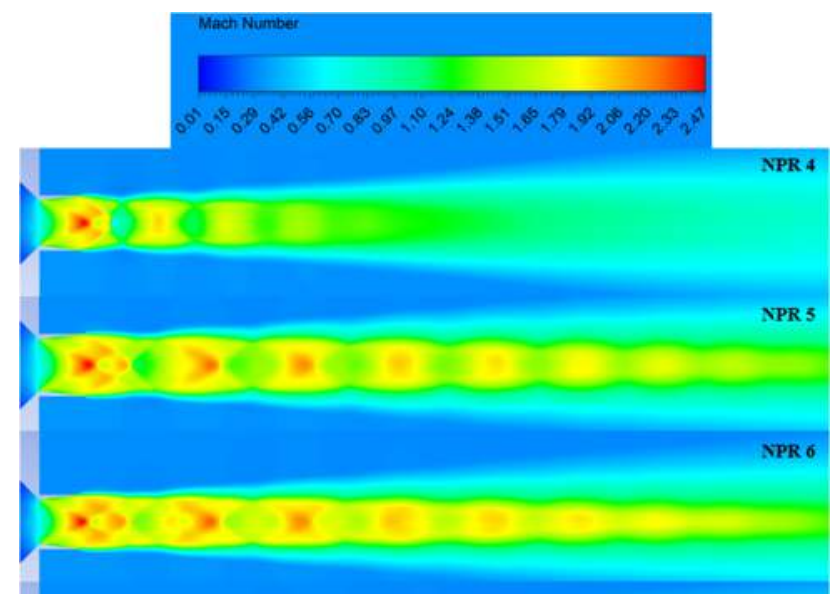

Fig. 11 Contours of Mach number with different NPRs for visualizing the shock cells.

The extent of jet mixing improves with the increase in the favorable pressure gradient at the nozzle exit. The Mach contour plots of the Mach 1.86 jet at NPRs 4, 5 and 6 are shown in Fig. 10. It is seen that, the number of shock cells increase with the increase in the NPR. Also, it is evident from the Mach contour plots that, the degree of jet mixing increases with the increase in the NPR or the favorable pressure gradient at the nozzle exit. The gradient of the characteristic decay, as shown in the plot for centerline pressure decay (Fig. 9), is highest for the NPR 6. This clearly demonstrates that, with the increasing NPR, the rate of jet mixing enhances considerably. In another words it can be inferred that, with the increasing NPR, the jet velocity increases downstream of the nozzle exit. This leads to the enhanced rate with which the vortices formed at the jet boundary reach to the jet centerline causing rapid decay of the jet.

\section{Conclusions}

The present work focuses in simulating the mixing characteristics of the Mach 1.86 jet at the over expanded condition for different NPRs in the range of 4 to 6 , with the step size of 1 . The results are presented in the form of centerline pressure decay along the jet centerline. The centerline pressure decay is the plot of non-dimensional total pressure along the jet centerline, with respect to the non-dimensional distance along the jet centerline. It is found that,

a) The grid becomes independent above the grid size of 223581 and thus, it is chosen as the grid for the present study so as to attain computational economy.

b) The number of shock cells increase with the increasing NPR and consequently with the decreasing level of adverse pressure gradient at the nozzle exit.

c) The core length of the jet increases with the increasing NPR which is because of the decreasing level of adverse pressure gradient at the nozzle exit.

d) The characteristic decay zone, which begins immediately after the potential core region and extends to the point along the jet centerline from which the jet attains self-similar profile, shortens with the increase in the NPR.

e) The gradient of the characteristic decay zone increases with the increasing NPR which might be attributed to the enhanced rate with which the viscous activity reaches the jet centerline, consequently affecting the jet centerline velocity.

f) The jet attains self-similar profile beyond $X / D=20$, which clearly demonstrates that the viscous activity which originated at the jet boundary reaches the jet centerline.

\section{References}

[1] Rathakrishan, E. Applied Gas Dynamics. Wiley India Pvt. Ltd., (2010).

[2] Hussain A.K.M.F. Coherent Structures and Turbuelnce. Journal of Fluid Mechanics, Volume 173, (1986), pp. 303-356.

[3] Shirie, J.W., and Seubold, J.G. "Length of the Supersonic core in High-Speed Jets. AIAA Journal, Volume 5(11), (1967), pp. 2062-2064.

[4] Ho, Chih-Ming., and Gutmark, E. Vortex induction and mass entrainment in a small-aspect-ratio elliptic jet. Journal of Fluid Mechanics, Volume 179, (1987), pp. 383-405.

[5] Gutmark, E., and Wygnanski, I. The planar turbulent jet. Journal of Fluid Mechanics, Volume 73(3), (1976), pp. 465-495.

[6] Launder, B.E., and Spalding, D.B. The Numerical Computation of Turbulent Flows. Computer Methods in Applied Mechanics and Engineering, Volume 3, (1974), pp. 269-289.

[7] Dash, S.M., Kenzakowski, D.C., Seiner, J.M., and Bhat, T.R.S. Recent Advances in Jet Flow Field Simulation: Part I - Steady Flow. 15 $5^{\text {th }}$ AIAA 
Aeroacoustics Conference, (1993, October 25-27), Long Beach, CA.

[8] Thies, Andrew T., and Tam, Christopher K.W. Computation of Turbulent Axisymmetric and Nonaxisymmetric Jet Flows Using the $\mathrm{k}-\varepsilon$ Model. AIAA Journal, Volume 34 (2), (1996), pp. 309-316.

[9] Evgenevna, Ilina E., Evgenevna, Ilina T., and Viktorovich, Butal P. Analysis of the Application of Turbulence Models in the Calculation of Supersonic Gas Jet. American Journal of Applied Science, Volume 11(11), (2014), pp. 1914-1920.

[10] Shantanu, Srivastava., and E., Rathakrishnan. Performance of Corrugated Limiting $\mathrm{Tab}$ in the Presence of Sharp Corners. International Review of Aerospace Engineering. Volume 7, No. 1, (2014), pp. 1-7.

[11] Anderson, J., Degrez, G., Degroote, J., Dick, E., Grundmann, R. and Virendeels, J. Computational Fluid Dynamics: An Introduction. Springer Verlag and Academic Press, $3^{\text {rd }}$ edition, (2009), Berlin, Heidelberg.

[12] Mohamad Nur Hidayat Mat., and Norzelawati Asmuin. Optimum Design of Nozzle Geometry of Dry Ice Blasting Using CFD for the Reduction of Noise Emission. International Journal of Integrated Engineering, Volume 10, No. 5, (2018), pp. 130-135.

[13] Wilcox, David C. Turbulence Modeling for CFD. DCW Industries, Inc., (1994), California.

[14] Azamahani Sadikin., Nurul Akma Mohd Yunus., Saiful Anuar Abd Hamid., Al Emaran Ismail., Salihatun Salleh., Sufizar Ahmad., Mohd Nasrull Abdol Rahman., Shahruddin Mahzan., and Sallehuddin Shah Ayop. A Comparative Study of Turbulence Models on Aerodynamics Characteristics of NACA0012 Airfoil. International Journal of Integrated Engineering, Volume 10, No. 1, (2018), pp. 134-137. 